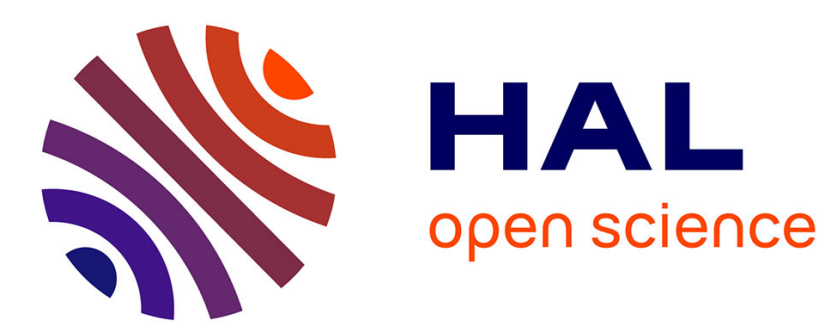

\title{
Band Structure of Crystals with Periodically Loaded Metallic Wires
}

\author{
Halim Boutayeb, Tayeb Denidni
}

\section{To cite this version:}

Halim Boutayeb, Tayeb Denidni. Band Structure of Crystals with Periodically Loaded Metallic Wires. Antennas and Propagation Society International Symposium 2006, IEEE, Oct 2006, Albuquerque, United States. pp. 4501 - 4504, 10.1109/APS.2006.1711636 . hal-00149266

\section{HAL Id: hal-00149266 https://hal.science/hal-00149266}

Submitted on 25 May 2007

HAL is a multi-disciplinary open access archive for the deposit and dissemination of scientific research documents, whether they are published or not. The documents may come from teaching and research institutions in France or abroad, or from public or private research centers.
L'archive ouverte pluridisciplinaire HAL, est destinée au dépôt et à la diffusion de documents scientifiques de niveau recherche, publiés ou non, émanant des établissements d'enseignement et de recherche français ou étrangers, des laboratoires publics ou privés. 


\title{
Band Structure of Crystals with Periodically Loaded Metallic Wires
}

\author{
Halim Boutayeb* and Tayeb A. Denidni \\ INRS-EMT, University of Quebec, Montreal, Canada.
}

\section{Introduction}

The propagation of electromagnetic waves in periodic structures has received recently an important interest [1]. Potential applications have been suggested in microwave and antenna domains, such as suppressing surface waves [2], designing directive antennas [3], or creating controllable microwave components [4].

The propagation of waves in periodic structures is described by means of a band theory. Different methods have been proposed for computing the band structure of periodic structures, e.g., the average field method [6], the order- $N$ method [7], and the hybrid plane-wave-integral-equation method [8]. A particular interest has been given to the dispersion characteristics of periodic structure formed by infinitely long metallic wires [1-10]. The band structure of periodic materials with loaded wires has not been studied enough. These materials are interesting for designing reconfigurable microwave components. The band structure of the discontinuous wire medium for different wire diameters and lengths has been studied in [11] in order to design controllable crystals. However, the effects of the active element have not been taken into account. In [12], an analysis of the dispersion of crystals with loaded wires has been presented. However, in open literature, there is no parametrical study for showing the effect of the value of the active elements.

In this contribution, numerical results are presented for the pass-bands and stop-bands of these 3-D periodic structures, at normal incidence. To compute the propagation constant, a transmission line model is used, where a 2-D periodic structure (grid) of discontinuous wires is modelled by a T-circuit. The T-circuit parameters are written in terms of the S-parameters of the grid, computed rigourously using the FDTD method.

\section{Computation of the propagation constant}

An infinite 3-D periodic structure of perfect metallic wires shown in Fig. 1 is considered. Its parameters are the periods $P_{x}, P_{y}$ and $P_{z}$, the wire diameter $a$ and the width $w$. The propagation of the transverse electric field in $x$-direction is considered. To compute the propagation constant $\beta_{x}$, the transmission line model is used, where a 2-D periodic structure in $y$-direction (see Fig. 1) is modelled by a T-circuit [11]. The T-circuit parameters are written in terms of the S-parameters of the grid, computed rigourously using the FDTD method, where Floquet boundaries conditions and a thin mesh $(\triangle=$ Period/80) are used. Only the fundamental mode is considered, then the limitations $P_{y} \leq P_{x}, P_{x} \leq \lambda$ and $P_{z} \leq \lambda$ are used.

In a first approximation, an electronic switch can be simulated by an equivalent circuit including R-L-C elements. The inductive term L, which essentially represent the connection wires to the device, can be considered included in the metallic wire, then active device can be represented only by an R-C circuit [4].

For a parallel or a series combination of a capacitor, $\mathrm{C}$, a resistor, $\mathrm{R}$, and an inductance, L, we integrated a model in our FDTD code, based on the scheme introduced by Piket-May et al [13]. The R-C circuits are periodically distributed along the wires, 
which form the 2-D photonic lattice.

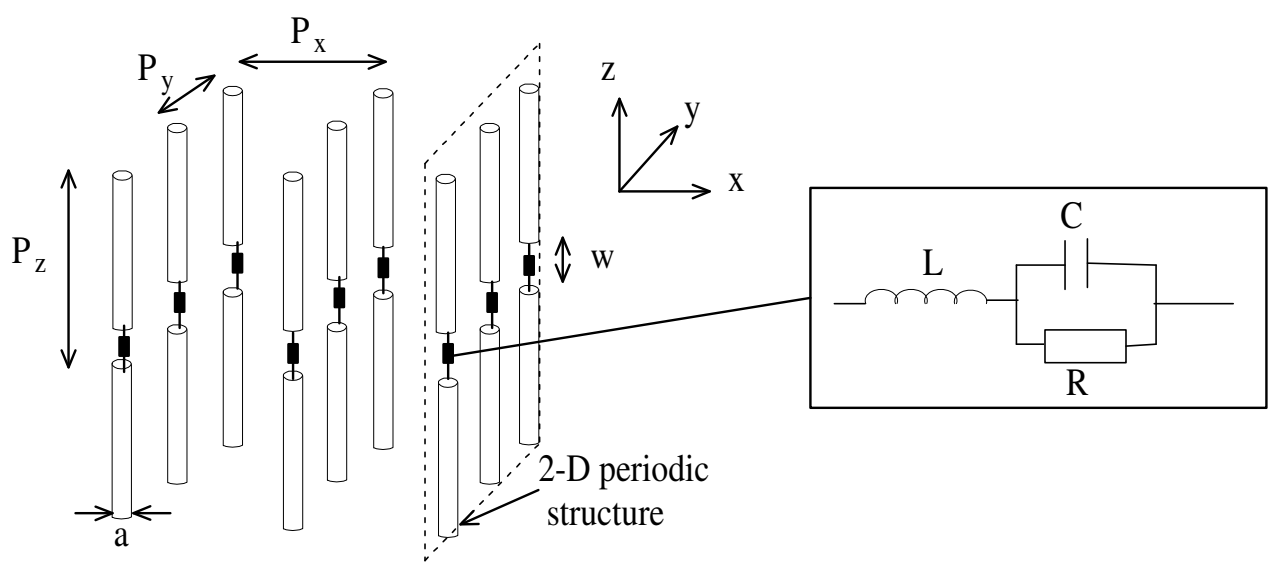

Figure 1: Infinite 3-D periodic structure of loaded metallic wires in air and equivalent RLC circuits for numerical simulations.

\section{Results and discussion}

The dual behavior in the pass-band and stop-band of the on-state and off-state structures is nearly obtained in the two first bands [11]. The limits of the two first bands of these structures are now studied for different wire diameters. We consider $P_{x}=P_{y}=P_{z}=P$. The R-C elements are chosen in agreement with characterization results obtained on high-speed commercial devices [4]. Based on practical considerations, we consider, for the on-state, $R=10 \Omega$; for off-state, we consider $R=30 \mathrm{k} \Omega$. Three capacitance values are chosen: $C=150 \mathrm{fF}, 30 \mathrm{fF}$ and $13 \mathrm{fF}$.

Fig. 2 presents the limits of the two first bands for the on-state case, and for the continuous-wire structure, versus the fill factor $a / P$. From this figure, it can be seen that the active element has less influence when the wire diameter is small. This is due to the fact that, for large diameter wires, the contrast between the thickness of the wire and the thickness of the active element is more important.

Fig. 3 presents the limits of the two first bands for the off-state case, for the continuous-wire structure, and for the discontinuous-wire structure, versus the fill factor $a / P$. According to this figure, compared to the discontinuous wire case, the active element have effect on small diameter wires and has no influence on large diameter wires. In addition, it can be also observed that for small diameter wires, the increase of the capacitance has the same effect that the increase of the width between wires for the discontinuous-wire case [11].

\section{Conclusion}

In this paper, the band structure for normal propagation of crystal formed by periodically loaded metallic wires has been analyzed for different wire diameters and for different values of the load, which are assimilated as diodes. The diodes have been simulated by an equivalent R-C circuit, which has been chosen in agreement with characterization results obtained with high-speed commercial devices. The influences of the values of the $\mathrm{R}$-C elements on on-state and off-state have been analyzed and the results have been compared to the previous results presented for continuous and 


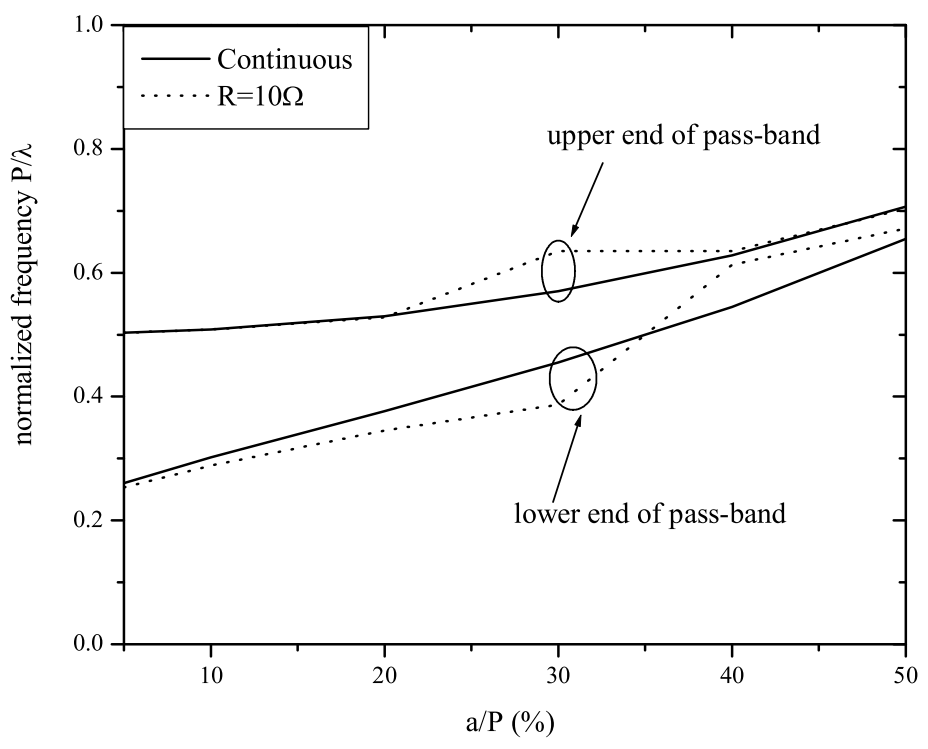

Figure 2: Two first bands limits for structures with continuous wires an for wires periodically loaded with $R=10 \Omega$ wires versus fill factor $a / P$.

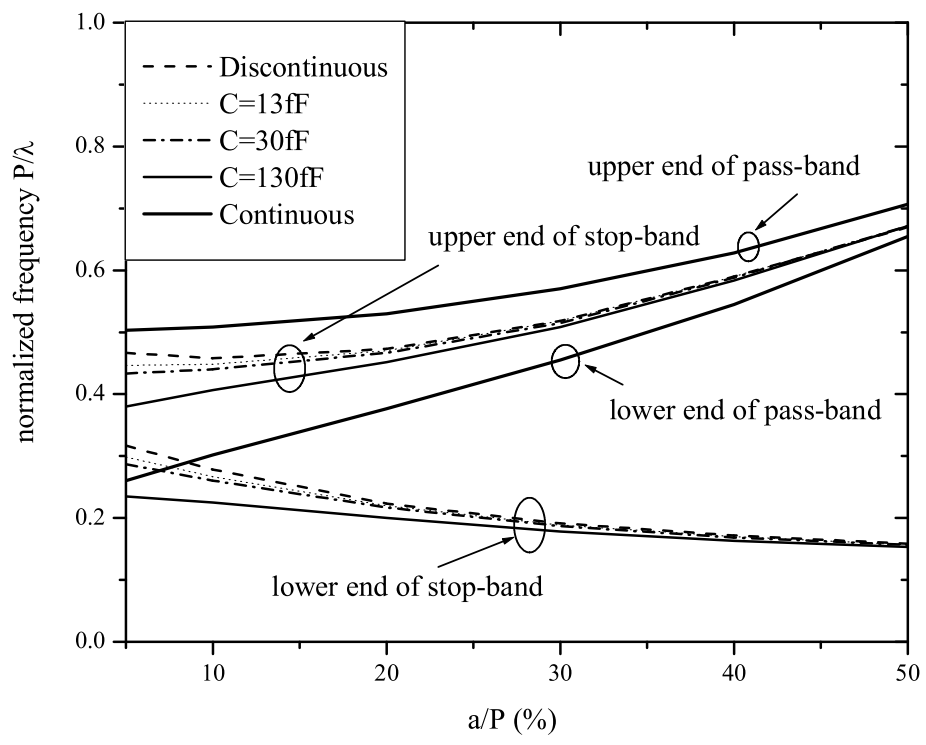

Figure 3: Two first bands limits for structures with continuous, discontinuous, and loaded wires versus fill factor $a / P$, with $R=30 k \Omega$, for different values of $C$.

discontinuous-wire structures. A potential application of this work is the design of controllable antennas. 


\section{References}

[1] K. Sakoda, Optical Properties of Photonic Crystal, ser. Opt. Sci. Berlin, Germany: Springer, 2001, vol. 80.

[2] F. Yang and Y. Rahmat-Samii, "Microstrip antennas integrated with electromagnetic bandgap (EBG) structures: a low mutual coupling design for array applications", IEEE Trans. on Antennas and Propagation, vol. 51, pp. 2936-2946, Oct. 2003.

[3] M. Thevenot, C. Cheype, A. Reineix and B. Jecko, "Directive Photonic Band-Gap Antennas", IEEE Trans. Microwave Theory Tech., vol. 47,pp. 2115-2122, Nov. 1999.

[4] J.M. Lourtioz, A. De Lustrac, F. Gadot, S. Rowson, A. Chelnokov, T. Brillat, A. Ammouche, J. Danglot, O. Vanbesien, and D. Lippens, "Toward controllable photonic crystals for centimeter and millimeter wave devices", Journal of Lightwave Technology, vol. 17, pp. 2025-2031, Nov. 1999.

[5] T. K. Chang, R. J. Langley, and E. A. Parker, "An active square loop frequency selective surfaces" IEEE Microwave Guided Wave Lett., vol. 3, no. 10, pp. 387388, 1993;

[6] C.R. Simovski, M. Qiu and S. He, "The average field approach for obtaining the band structure of crystals with conducting wire inclusions", Journal of Electromag. Waves and Appl., vol. 14, pp.449-468, 2000.

[7] C.T. Chan, Q.L. Yu, and K.M. Ho, "Order-N method spectral method for electromagnetic waves", Phys. Rev. B, Condens. Matter, vol. 51, pp. 16635-16642, 1995.

[8] M. Silveirinha and C. A. Fernandes, "A Hybrid method for the efficient calculation of the band structure of 3D-metallic crystal", IEEE Trans. Microw. Theory and Techn., vol. 52, pp. 889-902, March 2004.

[9] S. Tretyakov, Analytical modeling in applied electromagnetics, Artech House, INC., Norwood, 2003.

[10] C. A. Moses and N. Engheta, "Electromagnetic wave propagation in the wire medium: a complex medium with long thin inclusions", Wave Motion, vol. 34, pp. 301-317, Sept. 2001.

[11] H. Boutayeb, T. A. Denidni, A. Sebak and L. Talbi, "Band structure analysis of crystals with discontinuous metallic wires", IEEE Microwave Wireless Comp. Lett., vol.15, pp. 484-486, July 2005.

[12] P. A. Belov, C. R. Simovski, and S. A. Tretyakov, "Two-dimensional electromagnetic crystals formed by reactively loaded wires", Phys. Rev. E, vol. 66, p. 036610.1-036610.7, 2002.

[13] M. Piket-May, A. Taflove and J. Baron, "FD-TD modeling of digital signal propagation in 3-D circuits with passive and active loads ", IEEE Transactions on Microwave Theory and Techniques, vol. 42, pp. 1514 - 1523, Aug. 1994. 